\title{
Dynamic Analysis of Priority with Linear Decreasing Function of a Priority in the Service System with Four Types of Application
}

\author{
Fathollah Dadgar Arablou \\ Baku state University, AZ 1148, \\ Baku, Av. Academy of $Z$. \\ Khalilova 23
}

\begin{abstract}
The problem of choosing optimal parameters for dynamic priorities in the case of the various types of service requests with linearly decreasing function of priorities, performed experiments and obtained results. These results can be used to develop algorithms of functioning nodes for data and their software. In this paper we propose a method for solving the problem of choosing the optimal values of these coefficients that minimize the total value of the queue length in systems with four types of applications.
\end{abstract}

\section{General Terms}

Dynamic Analysis, Priority in the Service

\section{Keywords}

Service, dynamic priorities, Optimization, Dynamic priorities, Priority.

\section{INTRODUCTION}

As it is known to meet specified levels of quality of service (Quality of Service) in switches high-speed multimedia networks with a total buffer space consisting of (Shared Buffering) with a number of spatial priorities (Space Priorities), objectified making procedures in different types of applications uses a buffer of time priority (Time priorities), defining the rules for selecting the type and appearance of the $s$ buffer [1]. Thus in the classical scheme of priority service, it is assumed that a certain type of application compared to other bids at Go type have a higher priority simultaneously both species. The analysis shows that known in a very small amount of work, the cat on the ryh considered schemes with different levels of spatial priorities for sound control in lyayuschie intensities (probably) different types of applications, and loss of time, etc., and priorities affecting the time of their $s$ and delay (latency) in the buffer. In [5-7] proposed various schemes of spatial definition of priorities in order to find the optimal (in some sense) the size used in Buffer Memory in the nodes of different service networks. In [5] based on the actual working conditions of the first highspeed multimedia service networks as temporary priority is proposed to use the dynamic priorities change over time. In [5] it is noted that one of the circumstances restraining widely used in dynamic priorities changing over time is the absence of act of determining the coefficients determining the rate of change of the priority application of a certain type at its Research Institute and Waiting in the queue. The present work is one of the ways of summarizing research on the procedures developed for the solution of the issues for the case of an arbitrary number for types of applications. In systems with dynamic priorities decision on determining the type of application chosen on the service depends on the value of a function determined by the instantaneous priority application index type when where many types of applications. This function can be determined analytically by various methods. Thus, the function of a multiplicative nature defined in [8], where a coefficient that determines the rate of change of the priority application type when it is waiting in the queue; random latency applications type of admission to the current moment t. Investigation of dynamic priorities, timedependent, especially important in the organization of the service applications with limited waiting time [5], as well as in systems with finite-aging applications [7]. One of the aforementioned problems is the lack of methods for determining the coefficients in the above formula function priority however, that by proper selection of these factors can increase the efficiency of the system with respect to the selected quality criterion.

\section{DESCRIPTION OF THE MODEL WITH DYNAMIC PRIORITIES}

We consider a single-channel system in which to service requests received Poisson flows, and the intensity of the flow is and the time of service applications of this type has an exponential distribution which means The system unserviced application form unlimited queue buffer memory. Priorities service applications vary depending on the length of waiting in line, ie Select the type of application for the service is carried out based on the current values of the priority for each application depending on the time of her waiting in the queue. This service applications is carried out in order of relative priority, ie at the time of release to service channel enters the application, which has at any given time a maximum value of the function of the priority number of entities in the buffer memory. Here we investigate the case the relative priorities of the linearly decreasing function of priority applications in the system. In this case the function of the priority of applications decreases linearly when waiting in the queue, ie, factors determining the rate of decay of the priority application, is negative Note that such a situation i s typical in particular for networks with rapidly aging information whose value to the functioning of the network is reduced proportionally to the time of the delay before serving. In this case also, the choice of the number of requests waiting in the queue is carried out, so that the service application is received, for which, at the moment of release prioritization computer function has a maximum value. In this case, the waiting time in the queue of applications type are defined in [8]: 


$$
\tau_{q_{i}}=\frac{\tau_{q_{0}}+\sum_{j=1}^{i-1} \rho_{j} \tau_{q_{j}}\left(1-\frac{b_{j}}{b_{i}}\right)}{1-\sum_{j=1}^{i} \rho_{j}-\sum_{j=i+1}^{n} \rho_{j} \frac{b_{i}}{b_{j}}}
$$

Meaning depends only on the ratio of the parameters. When $\mathrm{i}=4$ :

$$
\begin{aligned}
& \tau_{q_{1}}=\tau_{q_{0}} /\left(1-\rho_{1}-\sum_{j=2}^{4} \rho_{j} b_{1} / b_{j}\right) \\
& \tau_{q_{2}}=\frac{\tau_{q_{0}}+\rho_{1} \tau_{q_{1}}\left(1-b_{1} / b_{2}\right)}{1-\rho_{1}-\rho_{2}-\sum_{j=3}^{4} \rho_{j} b_{2} / b_{j}} \\
& \tau_{q_{3}}=\frac{\tau_{q_{0}} /(1-\rho)-\sum_{j=3}^{4} \rho_{j} \tau_{q_{j}}\left(1-b_{j} / b_{2}\right)}{1-\rho_{1}-\rho_{2}-\rho_{3}-\rho_{4} b_{3} / b_{4}} \\
& \tau_{q_{4}}=\frac{\tau_{q_{0}}+\sum_{j=1}^{3} \rho_{j} \tau_{q_{j}}\left(1-b_{j} / b_{4}\right)}{1-\sum_{j=1}^{4} \rho_{j}}
\end{aligned}
$$

\section{OPTIMAL DYNAMIC ASSIGNMENT OF PRIORITIES}

Here we consider the problem of choosing the optimal values of coefficient in the case of four types of orders $(n=4)$. At the same time as the optimality criterion is chosen the total length of the queue of different types of applications, in other words, it is required to find such (optimal) values to the total length of the queue was minimal when set limits on the time-out each type of application. The problem is mathematically written as:

$L_{q}=\sum_{i=1}^{4} \lambda_{1} \tau_{q_{1}} \rightarrow \min$

With restrictions

$\tau_{q_{1}} \leq \tau_{q_{1}}^{*}, \quad \tau_{q_{2}} \leq \tau_{q_{2}}^{*}, \quad \tau_{q_{3}} \leq \tau_{q_{3}}^{*}, \quad \tau_{q_{4}} \leq \tau_{q_{4}}^{*}$

Where $\tau_{q_{1}}^{*}, \tau_{q_{2}}^{*}, \tau_{q_{3}}^{*}$ and $\tau_{q_{4}}^{*}$ are known quantities.

The problem (2) - (3) is as follows:

$$
\begin{aligned}
& L_{q}=\frac{\tau_{q_{0}}}{1-\rho} \cdot \frac{\left(\lambda_{1}-\rho \lambda_{1}+\lambda_{2}\right) b_{2}-\left(\rho_{2} \lambda_{2}+\rho_{1} \lambda_{2}\right) b_{1}}{\left(1-\rho_{1}\right) b_{2}-\rho_{2} b_{1}}+ \\
& \frac{\tau_{q_{0}} /(1-\rho)-\sum_{j=3}^{4} \rho_{j} \tau_{q_{j}}\left(1-b_{j} / b_{2}\right)}{1-\rho_{1}-\rho_{2}-\rho_{3}-\rho_{4} b_{3} / b_{4}} \lambda_{3}+
\end{aligned}
$$

$$
+\frac{\tau_{q_{0}}+\sum_{j=1}^{3} \rho_{j} \tau_{q_{j}}\left(1-b_{j} / b_{4}\right)}{1-\sum_{j=1}^{4} \rho_{j}} \lambda_{4} \rightarrow \min
$$

with restrictions

$$
\begin{aligned}
& \frac{\tau_{q_{0}} b_{2}}{\left(1-\rho_{1}\right) b_{2}-\rho_{2} b_{1}} \leq \tau_{q_{1}}^{*} \\
& \frac{\tau_{q_{0}}}{1-\rho} \cdot \frac{b_{2}-\left(\rho_{2}-\rho_{1}\right) b_{1}}{\left(1-\rho_{1}\right) b_{2}-\rho_{2} b_{1}} \leq \tau_{q_{2}}^{*} \\
& \frac{\tau_{q_{0}} /(1-\rho)-\sum_{j=3}^{4} \rho_{j} \tau_{q_{j}}\left(1-b_{j} / b_{2}\right)}{1-\rho_{1}-\rho_{2}-\rho_{3}-\rho_{4} b_{3} / b_{4}} \leq \tau_{q_{3}}^{*} \\
& \frac{\tau_{q_{0}}+\sum_{j=1}^{3} \rho_{j} \tau_{q_{j}}\left(1-b_{j} / b_{4}\right)}{1-\sum_{j=1}^{4} \rho_{j}} \leq \tau_{q_{4}}^{*}
\end{aligned}
$$

The problem (4) - (8) is a linear-fractional programming (ZLP). Using the methods of DLP these tasks can, reduced to the basic problem of linear programming (OZLP). To this end, we introduce new variables, $x_{j}=x_{0} b_{j}, y_{j}=y_{0} b_{j}$, $i=1,2$

Follows: $x_{0}=\left[(1-\rho)\left[\left(1-\rho_{1}\right) b_{1}+\rho_{1} b_{2}\right]\right]^{-1}$ and $y_{0}=\left[(1-\rho)\left[\left(1-\rho_{1}\right) b_{2}-\rho_{2} b_{1}\right]\right]^{-1}$

The problem (4) - (8) leads to the following OZLP:

$L_{q}=-\tau_{q_{0}}\left[\left(\lambda_{1}-\rho \lambda_{1}+\lambda_{2}\right) y_{2}-\left(\lambda_{2} \rho_{2}+\lambda_{2} \rho_{1}\right) y_{1}\right] \longrightarrow \max$

With restrictions:

$$
\begin{gathered}
(1-\rho) y_{2}+y_{3}=\frac{\tau_{q_{1}}^{*}}{\tau_{q_{0}}} \\
y_{2}-\left(\rho_{2}-\rho_{1}\right) y_{1}+y_{4}=\frac{\tau_{q_{2}}^{*}}{\tau_{q_{0}}} \\
y_{1}+y_{2}=1 \\
y_{1}, y_{2}, y_{3}, y_{4} \geq 0
\end{gathered}
$$


Solutions of (9) - (13) can be obtained by using artificial bases. This extended problem for (9) - (13) will be as follows:

$L_{q}=-\tau_{q_{0}}\left[\left(\lambda_{1}-\rho \lambda_{1}+\lambda_{2}\right) y_{2}-\left(\lambda_{2} \rho_{2}+\lambda_{2} \rho_{1}\right) y_{1}\right] \longrightarrow \max$

with restrictions:

$$
\begin{gathered}
(1-\rho) y_{2}+y_{3}=\frac{\tau_{q_{1}}^{*}}{\tau_{q_{0}}}, \\
y_{2}-\left(\rho_{2}-\rho_{1}\right) y_{1}+y_{4}=\frac{\tau_{q_{2}}^{*}}{\tau_{q_{0}}} \\
y_{1}+y_{2}+y_{5}=1, \text {, где } y_{j} \geq 0, j=1,5 .
\end{gathered}
$$

\section{NUMERICAL RESULTS}

To study the optimal dynamic assignment of priorities in queuing systems with four types of applications in the linear decreasing function of priority in $\rho_{i}=\lambda_{i} / \mu_{i}=(0.5,0.6$, $0.7,0.8,0.9) \quad$ voluminous numerical experiments performed and the results (tab.1-18). These results can be used to study the nature of the change rates of the priority of different types of applications, depending on the changes in the values of

$\tau^{*}{ }_{q_{i}}, i=1,4$ and (total) of the linearly decreasing functions of priority.

Table 1. $\rho=0.9 ; \tau_{q 2}^{*}=0.01 ; \tau_{q 3}^{*}=0.03$

\begin{tabular}{|l|l|l|}
\hline$\tau_{q_{1}}{ }^{*}$ & $\frac{b_{1}{ }^{*}}{b_{2}{ }^{*}}$ & $L_{q}{ }^{*}$ \\
\hline 0.02 & 0.47 & 0.00010 \\
\hline 0.03 & 0.23 & 0.00121 \\
\hline 0.05 & 0.13 & 0.00031 \\
\hline 0.07 & 0.05 & 0.00096 \\
\hline 0.09 & 0.01 & 0.00350 \\
\hline
\end{tabular}

Table 2. $\rho=0.9221 ; \tau_{q_{2}}^{*}=0.01$;

\begin{tabular}{|l|l|l|}
\hline$\tau_{q_{1}}^{*}$ & $\frac{b_{1}{ }^{*}}{b_{3}{ }^{*}}$ & $L_{q}^{*}$ \\
\hline 0.02 & 9.01 & 0.00004 \\
\hline 0.03 & 7.20 & 0.00011 \\
\hline 0.05 & 5.40 & 0.00020 \\
\hline 0.07 & 2.66 & 0.00035 \\
\hline 0.09 & 0.88 & 0.00047 \\
\hline
\end{tabular}

$\tau_{q_{3}}^{*}=0.03 ; \tau_{q_{4}}^{*}=0.05 ; \frac{b^{*}{ }_{1}}{b^{*}{ }_{3}}=0.12 ; \frac{b^{*}{ }_{1}}{b^{*}{ }_{4}}=0.17 ;$

$b_{i}<0 \quad \tau_{q_{4}}^{*}=0.05 ; \frac{b^{*}{ }_{2}}{b^{*}{ }_{1}}=0.12 ; \frac{b^{*}{ }_{4}}{b^{*}{ }_{1}}=0.15$;

$b_{i}<0$

Table 3. $\rho=0.9 ; \tau_{q 2}^{*}=0.01 ; \tau_{q 3}^{*}=0.03$;

\begin{tabular}{|l|l|l|}
\hline$\tau_{q_{1}}^{*}$ & $\frac{b_{1}{ }^{*}}{b_{4}{ }^{*}}$ & $L_{q}^{*}$ \\
\hline 0.02 & 0.48 & 0.00001 \\
\hline 0.03 & 0.27 & 0.00011 \\
\hline 0.05 & 0.16 & 0.00031 \\
\hline 0.07 & 0.03 & 0.00094 \\
\hline 0.09 & 0.01 & 0.00180 \\
\hline
\end{tabular}

$$
\tau_{q_{4}}^{*}=0.05 ; \frac{b^{*}{ }_{3}}{b^{*}{ }_{1}}=0.12 ; \frac{b^{*}{ }_{4}}{b^{*}{ }_{1}}=0.17 ; b_{i}<0
$$

Table 4. $\rho=0.9 ; \tau_{q 2}^{*}=0.01 ; \tau_{q 3}^{*}=0.03$;

\begin{tabular}{|l|l|l|}
\hline$\tau_{q_{2}}^{*}$ & $\frac{b_{1}{ }^{*}}{b_{2}{ }^{*}}$ & $L_{q}^{*}$ \\
\hline 0.02 & 9.12 & 0.00024 \\
\hline 0.03 & 7.22 & 0.00035 \\
\hline 0.05 & 5.44 & 0.00051 \\
\hline 0.07 & 2.66 & 0.00084 \\
\hline 0.09 & 1.88 & 0.00124 \\
\hline
\end{tabular}

$\tau_{q_{4}}^{*}=0.05 ; \frac{b^{*}{ }_{2}}{b^{*}{ }_{3}}=0.12 ; \frac{b^{*}{ }_{2}}{b^{*}{ }_{4}}=0.16 ; b_{i}<0$

Table 5. $\rho=0.9 ; \tau_{q 2}^{*}=0.01 ; \tau_{q 3}^{*}=0.03$;

\begin{tabular}{|l|l|l|}
\hline$\tau_{q_{2}}{ }^{*}$ & $\frac{b_{2}{ }^{*}}{b_{3}{ }^{*}}$ & $L^{*}{ }^{*}$ \\
\hline 0.02 & 0.44 & 0.00002 \\
\hline 0.03 & 0.25 & 0.00014 \\
\hline 0.05 & 0.11 & 0.00027 \\
\hline 0.07 & 0.04 & 0.00094 \\
\hline 0.09 & 0.01 & 0.00270 \\
& & \\
\hline
\end{tabular}

$$
\tau_{q_{4}}^{*}=0.05 ; \frac{b^{*}{ }_{1}}{b^{*}{ }_{2}}=0.12 ; \frac{b^{*}{ }_{2}}{b^{*}{ }_{4}}=0.15 ; b_{i}<0
$$


Table 6. $\rho=0.9 ; \tau_{q 2}^{*}=0.01 ; \tau_{q 3}^{*}=0.03$;

\begin{tabular}{|l|l|l|}
\hline$\tau_{q_{2}}^{*}$ & $\frac{b_{2}{ }^{*}}{b_{4}{ }^{*}}$ & $L_{q}^{*}$ \\
\hline 0.02 & 9.11 & 0.00004 \\
\hline 0.03 & 7.22 & 0.00025 \\
\hline 0.05 & 5.44 & 0.00037 \\
\hline 0.07 & 2.66 & 0.00081 \\
\hline 0.09 & 0.88 & 0.00169 \\
\hline
\end{tabular}

$\tau_{q_{4}}^{*}=0.05 ; \frac{b_{1}^{*}}{b^{*}{ }_{2}}=0.12 ; \frac{b^{*}{ }_{2}}{b_{3}^{*}}=0.15 ; b_{i}<0$

Table 7. $\rho=0.9 ; \tau_{q 2}^{*}=0.01 ; \tau_{q 3}^{*}=0.03$;

\begin{tabular}{|l|l|l|}
\hline$\tau_{q_{3}}{ }^{*}$ & $\frac{b_{3}{ }^{*}}{b_{2}{ }^{*}}$ & $L_{q}{ }^{*}$ \\
\hline 0.02 & 0.04 & 0.00011 \\
\hline 0.03 & 0.09 & 0.00031 \\
\hline 0.05 & 0.15 & 0.00076 \\
\hline 0.07 & 0.29 & 0.00104 \\
\hline 0.09 & 0.55 & 0.00290 \\
& & \\
\hline
\end{tabular}

$\tau_{q_{4}}^{*}=0.05 ; \frac{b^{*}{ }_{4}}{b^{*}{ }_{2}}=0.12 ; \frac{b^{*}{ }_{3}}{b^{*}{ }_{4}}=0.17 ; b_{i}<0$

Table 8. $\rho=0.9 ; \tau_{q 2}^{*}=0.01 ; \tau_{q 3}^{*}=0.03$;

\begin{tabular}{|l|l|l|}
\hline$\tau_{q_{3}}^{*}$ & $\frac{b_{4}{ }^{*}}{b_{2}{ }^{*}}$ & $L_{q}^{*}$ \\
\hline 0.02 & 2.11 & 0.00012 \\
\hline 0.03 & 3.22 & 0.00035 \\
\hline 0.05 & 5.44 & 0.00056 \\
\hline 0.07 & 7.66 & 0.00071 \\
\hline 0.09 & 9.88 & 0.00094 \\
\hline
\end{tabular}

$\tau_{q_{4}}^{*}=0.01 ; \frac{b_{3}^{*}}{b^{*}{ }_{2}}=0.12 ; \frac{b^{*}{ }_{3}}{b^{*}{ }_{4}}=0.17 ; b_{i}<0$
Table 9. $\rho=0.9 ; \tau_{q 2}^{*}=0.01 ; \tau_{q 3}^{*}=0.03$;

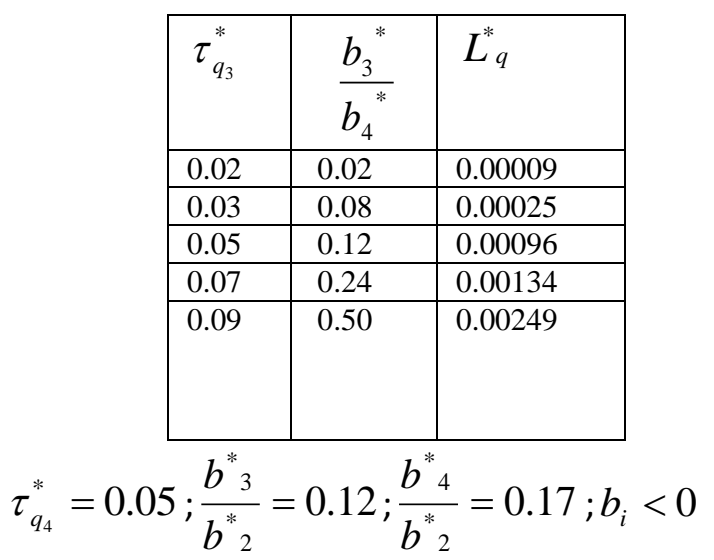

Table 10. $\rho=0.9 ; \tau_{q 2}^{*}=0.01 ; \tau_{q 3}^{*}=0.03$ ；

$$
\begin{gathered}
\begin{array}{|l|l|l|}
\hline \tau_{q_{4}}^{*} & \frac{b_{1}{ }^{*}}{b_{4}{ }^{*}} & L_{q}^{*} \\
\hline 0.02 & 8.11 & 0.00007 \\
\hline 0.03 & 7.22 & 0.00025 \\
\hline 0.05 & 5.44 & 0.00056 \\
\hline 0.07 & 2.66 & 0.00071 \\
\hline 0.09 & 0.88 & 0.00104 \\
\hline \multicolumn{2}{|c|}{b^{*}{ }_{2}^{*}} \\
\tau_{q_{4}}^{*}=0.05 ; \frac{b^{*}}{b^{*}{ }_{4}}=0.12 ; \frac{b^{*}{ }_{4}}{b^{*}}=0.16 ; b_{i}<0
\end{array}
\end{gathered}
$$

Table 11. $\rho=0.9 ; \tau_{q 2}^{*}=0.01 ; \tau_{q 3}^{*}=0.03$;

\begin{tabular}{|l|l|l|}
\hline$\tau_{q 4}^{*}$ & $\frac{b_{2}{ }^{*}}{b_{4}{ }^{*}}$ & $L_{q}^{*}$ \\
\hline 0.02 & 0.44 & 0.00008 \\
\hline 0.03 & 0.25 & 0.00041 \\
\hline 0.05 & 0.11 & 0.00086 \\
\hline 0.07 & 0.04 & 0.00137 \\
\hline 0.09 & 0.01 & 0.00270 \\
& & \\
\hline
\end{tabular}

$\tau_{q_{4}}^{*}=0.05 ; \frac{b^{*}{ }_{2}}{b^{*}{ }_{4}}=0.12 ; \frac{b^{*}{ }_{3}}{b^{*}{ }_{4}}=0.16 ; b_{i}<0$ 
Table 12. $\rho=0.9 ; \tau_{q 2}^{*}=0.01 ; \tau_{q 3}^{*}=0.03$;

\begin{tabular}{|l|l|l|}
\hline$\tau_{q_{4}}^{*}$ & $\frac{b_{3}{ }^{*}}{b_{4}{ }^{*}}$ & $L_{q}^{*}$ \\
\hline 0.02 & 9.10 & 0.00001 \\
\hline 0.03 & 7.21 & 0.00025 \\
\hline 0.05 & 5.45 & 0.00039 \\
\hline 0.07 & 2.69 & 0.00057 \\
\hline 0.09 & 1.08 & 0.00108 \\
\hline
\end{tabular}

$\tau_{q_{4}}^{*}=0.04 ; \frac{b_{1}^{*}}{b^{*}{ }_{4}}=0.12 ; \frac{b^{*}{ }_{3}}{b_{4}{ }_{4}}=0.17 ; b_{i}<0$

Table 13. $\tau_{q_{1}}^{*}=0.01 ; \tau_{q 2}^{*}=0.03 ; \tau_{q 3}^{*}=0.05$;

\begin{tabular}{|l|l|l|}
\hline$\rho$ & $\frac{b_{1}{ }^{*}}{b_{2}{ }^{*}}$ & $L_{q}{ }^{*}$ \\
\hline 0.5 & 0.54 & 0.00004 \\
\hline 0.6 & 0.21 & 0.00031 \\
\hline 0.7 & 0.14 & 0.00076 \\
\hline 0.8 & 0.05 & 0.00094 \\
\hline 0.9 & 0.01 & 0.00255 \\
\hline
\end{tabular}

$\tau_{q_{4}}^{*}=0.06 ; \frac{b^{*}{ }_{1}}{b^{*}{ }_{3}}=0.10 ; \frac{b^{*}{ }_{1}}{b^{*}{ }_{4}}=0.12 ; \frac{b^{*}{ }_{2}}{b^{*}{ }_{3}}=0.14 ;$

$\tau_{q_{4}}^{*}=0.05 ; \frac{b^{*}{ }_{1}}{b^{*}{ }_{2}}=0.10 ; \frac{b^{*}{ }_{1}}{b^{*}{ }_{4}}=0.12 ; \frac{b^{*}{ }_{2}}{b^{*}{ }_{3}}=0.13 ;$

Table 14. $\tau_{q_{1}}^{*}=0.01 ; \tau_{q 2}^{*}=0.03 ; \tau_{q 3}^{*}=0.04$ ；

\begin{tabular}{|l|l|l|}
\hline$\rho$ & $\frac{b_{1}{ }^{*}}{b_{3}{ }^{*}}$ & $L_{q}$ \\
\hline 0.5 & 9.15 & 0.00002 \\
\hline 0.6 & 7.24 & 0.00025 \\
\hline 0.7 & 5.47 & 0.00046 \\
\hline 0.8 & 2.68 & 0.00081 \\
\hline 0.9 & 0.08 & 0.00124 \\
\hline
\end{tabular}

$\frac{b^{*}{ }_{2}}{b^{*}{ }_{4}}=0.15 ; \frac{b_{3}^{*}}{b^{*}{ }_{2}}=0.16 ; \frac{b^{*}{ }_{4}}{b^{*}{ }_{2}}=0.17 ; \frac{b^{*}{ }_{3}}{b^{*}{ }_{4}}=0.16$

$; \frac{b^{*}{ }_{2}}{b^{*}{ }_{4}}=0.14 ; \frac{b^{*}{ }_{3}}{b_{2}{ }_{2}}=0.15 ; \frac{b^{*}{ }_{4}}{b^{*}{ }_{2}}=0.17 \frac{b^{*}{ }_{3}}{b^{*}{ }_{4}}=0.16$

$b_{i}<0 \quad b_{i}<0$
Table 15. $\tau_{q_{1}}^{*}=0.01 ; \tau_{q 2}^{*}=0.03 ; \tau_{q 3}^{*}=0.05$;

\begin{tabular}{|l|l|l|}
\hline$\rho$ & $\frac{b_{1}{ }^{*}}{b_{4}{ }^{*}}$ & $L_{q}^{*}$ \\
\hline 0.5 & 0.49 & 0.00010 \\
\hline 0.6 & 0.28 & 0.00031 \\
\hline 0.7 & 0.14 & 0.00076 \\
\hline 0.8 & 0.06 & 0.00134 \\
\hline 0.9 & 0.01 & 0.00260 \\
& & \\
\hline
\end{tabular}

$\tau_{q_{4}}^{*}=0.06 ; \frac{b^{*}{ }_{1}}{b^{*}{ }_{2}}=0.10 ; \frac{b_{1}^{*}{ }_{1}}{b_{3}{ }_{3}}=0.12 ; \frac{b^{*}{ }_{2}}{b^{*}{ }_{3}}=0.13$;

$\tau_{q_{4}}^{*}=0.05 ; \frac{b^{*}{ }_{1}}{b^{*}{ }_{2}}=0.10 ; \frac{b^{*}{ }_{1}}{b^{*}{ }_{3}}=0.12 ; \frac{b^{*}{ }_{1}}{b^{*}{ }_{4}}=0.13$;

Table 16. $\tau_{q_{1}}^{*}=0.01 ; \tau_{q 2}^{*}=0.03 ; \tau_{q 3}^{*}=0.04$;

\begin{tabular}{|l|l|l|}
\hline$\rho$ & $\frac{b_{2}{ }^{*}}{b_{3}{ }^{*}}$ & $L_{q}{ }^{*}$ \\
\hline 0.5 & 10.01 & 0.00009 \\
\hline 0.6 & 8.21 & 0.00037 \\
\hline 0.7 & 5.46 & 0.00046 \\
\hline 0.8 & 4.63 & 0.00061 \\
\hline 0.9 & 2.87 & 0.00097 \\
\hline
\end{tabular}

$\frac{b^{*}{ }_{2}}{b^{*}{ }_{4}}=0.14 ; \frac{b^{*}{ }_{3}}{b^{*}{ }_{2}}=0.17 ; \frac{b^{*}{ }_{4}}{b^{*}{ }_{2}}=0.16 ; \frac{b^{*}{ }_{3}}{b^{*}{ }_{4}}=0.15$

$; \frac{b^{*}{ }_{2}}{b^{*}{ }_{4}}=0.14 ; \frac{b^{*}{ }_{3}}{b^{*}{ }_{2}}=0.17 ; \frac{b^{*}{ }_{4}}{b^{*}{ }_{2}}=0.16 \frac{b^{*}{ }_{3}}{b^{*}{ }_{4}}=0.15$

$b_{i}<0 \quad b_{i}<0$

Table 17. $\tau_{q_{1}}^{*}=0.01 ; \tau_{q 2}^{*}=0.03 ; \tau_{q 3}^{*}=0.05 ;$

\begin{tabular}{|l|l|l|}
\hline$\rho$ & $\frac{b_{2}{ }^{*}}{b_{4}{ }^{*}}$ & $L_{q}{ }^{*}$ \\
\hline 0.5 & 0.47 & 0.00003 \\
\hline 0.6 & 0.28 & 0.00031 \\
\hline 0.7 & 0.12 & 0.00086 \\
\hline 0.8 & 0.05 & 0.00134 \\
\hline 0.9 & 0.02 & 0.00270 \\
& & \\
\hline
\end{tabular}


$\tau_{q_{4}}^{*}=0.04 ; \frac{b_{1}^{*}}{b^{*}{ }_{3}}=0.10 ; \frac{b^{*}{ }_{1}}{b^{*}{ }_{4}}=0.12 ; \frac{b^{*}{ }_{2}}{b^{*}{ }_{3}}=0.14$
$\tau_{q_{4}}^{*}=0.07 ; \frac{b_{1}^{*}}{b^{*}{ }_{2}}=0.10 ; \frac{b^{*}{ }_{1}}{b^{*}{ }_{4}}=0.12 ; \frac{b_{2}{ }_{2}}{b^{*}{ }_{3}}=0.15$

Table 18. $\tau_{q_{1}}^{*}=0.01 ; \tau_{q 2}^{*}=0.03 ; \tau_{q 3}^{*}=0.05$;

\begin{tabular}{|l|l|l|}
\hline$\rho$ & $\frac{b_{3}{ }^{*}}{b_{2}{ }^{*}}$ & $L_{q}{ }^{*}$ \\
\hline 0.5 & 2.11 & 0.00005 \\
\hline 0.6 & 3.22 & 0.00027 \\
\hline 0.7 & 5.44 & 0.00046 \\
\hline 0.8 & 7.66 & 0.00091 \\
\hline 0.9 & 9.88 & 0.00114 \\
\hline
\end{tabular}

$$
\begin{aligned}
& \frac{b^{*}{ }_{1}}{b^{*}{ }_{2}}=0.16 ; \frac{b_{3}^{*}}{b^{*}{ }_{2}}=0.17 ; \frac{b^{*}{ }_{4}}{b^{*}{ }_{2}}=0.15 ; \frac{b_{3}{ }_{3}}{b^{*}{ }_{4}}=0.14 \\
& ; \frac{b^{*}{ }_{2}}{b^{*}{ }_{4}}=0.16 ; \frac{b^{*}{ }_{1}}{b^{*}{ }_{3}}=0.13 ; \frac{b^{*}{ }_{4}}{b^{*}{ }_{2}}=0.17 \frac{b^{*}{ }_{3}}{b^{*}{ }_{4}}=0.15 \\
& b_{i}<0 \quad b_{i}<0
\end{aligned}
$$

Table 19. $\tau_{q_{1}}^{*}=0.01 ; \tau_{q 2}^{*}=0.03 ; \tau_{q 3}^{*}=0.05$;

\begin{tabular}{|l|l|l|}
\hline$\rho$ & $\frac{b_{4}{ }^{*}}{b_{2}{ }^{*}}$ & $L^{*}$ \\
\hline 0.5 & 0.01 & 0.00009 \\
\hline 0.6 & 0.04 & 0.00031 \\
\hline 0.7 & 0.11 & 0.00076 \\
\hline 0.8 & 0.25 & 0.00134 \\
\hline 0.9 & 0.44 & 0.00285 \\
& & \\
\hline
\end{tabular}

$\tau_{q_{4}}^{*}=0.04 ; \frac{b^{*}{ }_{1}}{b^{*}{ }_{3}}=0.10 ; \frac{b^{*}{ }_{1}}{b^{*}{ }_{4}}=0.12 ; \frac{b^{*}{ }_{2}}{b^{*}{ }_{3}}=0.13 ;$

$\tau_{q_{4}}^{*}=0.04 ; \frac{b^{*}{ }_{1}}{b^{*}{ }_{2}}=0.10 ; \frac{b^{*}{ }_{1}}{b^{*}{ }_{4}}=0.12 ; \frac{b^{*}{ }_{2}}{b^{*}{ }_{3}}=0.13 ;$

Table 20. $\tau_{q_{1}}^{*}=0.01 ; \tau_{q 2}^{*}=0.03 ; \tau_{q 3}^{*}=0.05$;

\begin{tabular}{|l|l|l|}
\hline$\rho$ & $\frac{b_{3}{ }^{*}}{b_{4}{ }^{*}}$ & $L_{q}^{*}$ \\
\hline 0.5 & 9.88 & 0.00008 \\
\hline 0.6 & 7.66 & 0.00024 \\
\hline 0.7 & 5.44 & 0.00039 \\
\hline 0.8 & 3.22 & 0.00055 \\
\hline 0.9 & 2.11 & 0.00089 \\
\hline
\end{tabular}

$\frac{b^{*}{ }_{2}}{b^{*}{ }_{4}}=0.14 ; \frac{b_{3}^{*}}{b^{*}{ }_{2}}=0.17 ; \frac{b_{1}^{*}}{b^{*}{ }_{2}}=0.16 ; \frac{b_{3}^{*}}{b^{*}{ }_{4}}=0.15$

$\frac{b^{*}{ }_{2}}{b^{*}{ }_{4}}=0.14 ; \frac{b^{*}{ }_{3}}{b^{*}{ }_{2}}=0.17 ; \frac{b_{4}^{*}}{b^{*}{ }_{2}}=0.16 \frac{b^{*}{ }_{1}}{b^{*}{ }_{3}}=0.15$

$b_{i}<0 \quad b_{i}<0$

It shows only the results of numerical experiments, for which the optimization problem are the best solutions. It is evident that under certain combinations of values of parameters of the system load $\left(\rho_{1}, i=\overline{1,5}\right)$ and upper bounds for the average waiting time of different types of applications $\tau^{*}{ }_{q i}, i=1,4$ corresponding optimization problem will not have a solution. Analysis of the results for the model with decreasing functions of priority to the following conclusions:

With increasing $\tau_{q_{4}}^{*}$ Decreases with increasing value ratios $b_{1}^{*} / b_{2}^{*}, b_{1}^{*} / b_{3}^{*}, b_{1}^{*} / b_{4}^{*}$;

With increasing $\tau_{q_{4}}^{*}$ Decreases with increasing value ratios $b_{1}^{*} / b_{2}^{*}, b_{2}^{*} / b_{3}^{*}, b_{2}^{*} / b_{4}^{*}$;

With increasing $\tau_{q_{3}}^{*}$ Decreases with increasing value ratios $b_{3}^{*} / b_{2}^{*}, b_{4}^{*} / b_{2}^{*}, b_{3}^{*} / b_{4}^{*}$;

With increasing $\tau_{q_{4}}^{*}$ Decreases with increasing value ratios $b_{1}^{*} / b_{4}^{*}, b_{2}^{*} / b_{4}^{*}, b_{3}^{*} / b_{4}^{*}$; with an increase in the total value of load increases and decreases relations $b_{3}^{*} / b_{2}^{*}$, $b_{4}^{*} / b_{2}^{*}$, value ratio $b_{1}^{*} / b_{2}^{*}, b_{1}^{*} / b_{3}^{*}, b_{1}^{*} / b_{4}^{*}, b_{2}^{*} / b_{3}^{*}$, $b_{2}^{*} / b_{4}^{*} b_{3}^{*} / b_{4}^{*}$;

\section{CONCLUSION}

This paper proposed computational procedure for finding the optimal values of the dynamic priorities, time-dependent applications which are waiting in the queue. The solution of these problems in the case of four types of applications is reduced to solving specific problems of fractional-linear programming. The results can be used in high-speed networks with heterogeneous applications. Currently, studies are conducted on the synthesis procedures developed to address the issues under consideration for any number of types of applications. 


\section{REFERENCES}

[1] Pitts J.M., Schormans J.A. Introduction to ATM design and performance. NY: John Wiley \& Sons., 1997.

[2] Chao H.J., Peckan I.H. Queue management with multiple delay and loss priorities for ATM switches // Proc. ICC'94,1994.-pp.1184-1189.

[3] Lee Y., Choi BDQueueing system with multiple delay and loss priorities for ATM networks // Information systems .2001.- vol. 138. - pp.7-29.

[4] Kim C.S., Melikov A.Z., Ponomarenko L.A. Approximation method for performance analysis of queuing system with multimedia traffics // Applied and Comput. Math.2007.-vol.6, no.2.- pp.218-226.

[5] Kim Ji Song, A.Z.Melikov, BG Ismailov Optimal dynamic assignment of priorities in queuing systems with two types of applications and Riga. AVT №6,2008, s57-65.

[6] Melikov AZ, Ismayilov BG Optimization of hierarchical structures of data networks // Math. ANAS, Series of Physical-Technical and Mathematical nauk.1997. №6 s.69-73.

[7] Ismayilov BG design a distributed network of service production facilities and gas treatment // $\mathrm{M} \therefore$ Gas promyshlennost.NTS. 2001, №1. s.29-35.

[8] Communication seti.- Kleinrock L. M .: Science, 1970.

\section{AUTHOR PROFILE}

Fathulah Dadgar Arablow: Ph.D. Student of computer science in Baku University, interested in multi-media and web mining, mobile ad hoc network and security, Distributed Systems. 